\title{
Democracia y guerra civil: ciudadanía y establecimiento de la paz en Costa de Marfil ${ }^{*}$
}

\author{
Autor \\ Abu Bakarr Bab* \\ TRADUCTOR \\ Julián López de Mesa** \\ Universidad Santo Tomás, Bogotá, Colombia \\ https://orcid.org/0000-0003-1922-5093
}

\section{RESUMEN}

La guerra civil en Costa de Marfil presenta características únicas en relación con las causas de las guerras civiles y la naturaleza de los procesos de paz en África occidental. Es un conflicto impulsado, en gran parte, por agravios políticos y sociales concretos sobre la ciudadanía. Además, está marcado por un esfuerzo significativo por parte de los beligerantes para apropiarse del proceso de paz y negociar directamente. Este artículo rastrea la guerra civil hasta la politización de la ciudadanía y el origen étnico durante el proceso de democratización. Sostiene que los acuerdos de paz elaborados por la comunidad internacional no lograron poner fin al conflicto porque se basaron, en gran medida, en las fórmulas de paz tradicionales y prestaron atención insuficiente al tema subyacente de la ciudadanía. En contraste, el acuerdo de paz forjado por los marfileños ha sido relativamente exitoso, porque abordó directamente

* Esta reimpresión traducida está disponible en acceso abierto solo con el permiso de Oxford University Press, en nombre de la Royal African Society. Para consultar y citar el artículo original: Bah, A. B. (2010). Democracy and civil war: Citizenship and peacemaking in Côte d'Ivoire. African Affairs, 109(437), 597-615. 10.1093/ afraf/adq046. (C) Abu Bakarr Bah (2010). Todos los derechos reservados. Para obtener permiso para reutilizar cualquier parte de este artículo, por favor escribir a: journals.permissions@oup.com

* Professor asociado de sociología de Northern Illinois University y editor jefe de African Conflict \& Peacebuilding Review. También es profesor asociado del Centro de Liderazgo y Desarrollo de ong. Es autor de Breakdown and Reconstitution: Democracy, the Nation-State and Ethnicity in Nigeria, y de numerosos artículos en revistas como Critical Sociology, African Affairs e International Journal of Politics, Culture, and Society. Actualmente investiga sobre guerras civiles y construcción de Estado en África occidental.

*** Docente de la Facultad de Gobierno y Relaciones Internacionales de la Universidad Santo Tomás, Bogotá, Colombia. 
el tema de la ciudadanía y restableció la propiedad nacional del proceso de paz. Este artículo se centra en el proceso de paz y la relación intrínseca entre la ciudadanía y el progreso hacia la paz en Costa de Marfil. Además, conecta el discurso sobre la democracia en África con el tema principal de la ciudadanía, y subraya la relación fluida entre ciudadanía y democracia en la política africana.

Palabras clave: acuerdos de paz, ciudadanía.

\begin{abstract}
The civil war in Côte d'Ivoire presents unique features with respect to the causes of civil wars and the nature of peace processes in West Africa. It is a conflict largely driven by concrete political and social grievances over citizenship. In addition, it is marked by a significant effort by the belligerents to take ownership of the peace process and negotiate directly. This article traces the civil war to the politicization of citizenship and ethnicity during the democratization process. It argues that the peace agreements engineered by the international community failed to end the conflict largely because they relied heavily on traditional peace formulas and paid insufficient attention to the underlying issue of citizenship. In contrast, the peace agreement forged by Ivorians has been relatively successful because it directly addressed the citizenship issue and restored domestic ownership of the peace process. This article focuses on the peace process and the intrinsic relation between citizenship and progress toward peace in Côte d'Ivoire. In addition, it connects the discourse on democracy in Africa with the salient issue of citizenship and underscores the fluidity of citizenship and democracy in African politics.
\end{abstract}

Keywords: peace agreement, citizenship

\title{
INTRODUCCIÓN
}

El conflicto en Costa de Marfil — que estalló después de las sangrientas guerras civiles en Liberia y Sierra Leona, y la violencia política masiva en Guinea Bissau- 
agregó un desafío de seguridad más para la comunidad internacional (Adeleke, 1995; Adebajo, 2002; Adebajo y Rashid, 2004). Aunque es tentador ver Costa de Marfil como simplemente otro caso de problemas en África occidental con el que la comunidad internacional ha tenido que lidiar, la guerra civil en este territorio se aleja significativamente de las modalidades de guerra y paz comúnmente encontradas en la región y exhibe dos características únicas.

Primero, la guerra de Costa de Marfil tiene una causa política clara que no se puede reducir a las ambiciones de un señor de la guerra. En Sierra Leona y Liberia, por ejemplo, las guerras civiles fueron impulsadas no solo por la mala gobernanza, sino también por las ambiciones de poder y riqueza que albergaban Charles Taylor y Foday Sankoh (Adebajo, 2001 y Richards, 2005). La paz solo llegó a estos países después de que los señores de la guerra fueron detenidos. El caso marfileño no encaja perfectamente con los argumentos comunes sobre la economía política de la guerra y los estados fallidos utilizados para explicar las guerras civiles africanas (Zartman, 1995; Reno, 1999; Collier y Hoeffler, 2004; Woods, 2003). En contraste, la guerra de Costa de Marfil se trata de reclamos de ciudadanía controvertidos (Dozon, 2000; Marshall-Fratani, 2006; Akokpari, 2008).

Una segunda característica distintiva es la propiedad interna del proceso de paz. Los recientes conflictos africanos, como las guerras en Liberia y Sierra Leona, han atraído importantes intervenciones internacionales para mejorar las condiciones humanitarias y restaurar la seguridad regional (Adebajo, 2001). Consciente de la centralidad económica de Costa de Marfil en la región, la comunidad internacional también se involucró (o se enredó) rápidamente en la resolución del conflicto.

Las mediaciones internacionales no necesariamente han llevado a una resolución rápida de los conflictos africanos (Goulding, 1999). Liberia y Sierra Leona languidecieron durante aproximadamente una década, mientras que la comunidad internacional hizo repetidos esfuerzos para negociar y hacer cumplir un acuerdo de paz duradero. Después de más de cuatro años de mediación internacional en Costa de Marfil, la paz siguió siendo difícil. La diferencia crítica es que, si bien las partes interesadas en Liberia y Sierra Leona no pudieron llegar a un acuerdo sin una fuerte 
participación de la comunidad internacional, los marfileños parecían dudar de la intervención internacional y sí estaban dispuestos a abordar sus propios problemas (Koulibaly, 2003; Smith, 2003; Epstein, 2008; entrevista a diplomático europeo, Abiyán, 6 de junio 2008; entrevista a diplomático africano, Abiyán, 7 de junio de 2008). Se comunicaron con sus compatriotas y forjaron el Acuerdo de Uagadugú, con una participación mínima de mediadores internacionales. Lo más importante es que el acuerdo abordó directamente el reclamo de ciudadanía subyacente.

El caso marfileño ofrece lecciones útiles para tratar los conflictos en África. Señala la importancia de la ciudadanía y los peligros de las políticas de identidad en las democracias africanas. Claramente, la política democrática requiere una clara definición de ciudadanía. El conflicto de Costa de Marfil también expone importantes deficiencias en la manera en que los procesos de paz impulsados internacionalmente tratan las causas subyacentes de los conflictos africanos. Si bien el tema de la ciudadanía era evidente en los discursos que rodean el conflicto y el proceso de paz, los acuerdos de mediación internacional no le prestaron suficiente atención. Este conflicto muestra que el reconocimiento temprano y la profunda apreciación de los reclamos subyacentes son fundamentales para forjar un acuerdo de paz duradero. También da fe de la importancia de incorporar mecanismos creativos en el acuerdo de paz para rectificar los errores que llevan a los reclamos. Los detalles de los acuerdos de paz mediados internacionalmente influyeron mucho en las modalidades de compartir el poder, el alto el fuego y las elecciones, dejando de lado la creación de un mecanismo para resolver el problema de la ciudadanía.

Este artículo examina la manera en que la ciudadanía degeneró en un importante problema político en la lucha por la democracia después de la muerte del presidente Félix Houphouët-Boigny en 1993, y la manera en que se trató este tema en sucesivos acuerdos de paz. Se considera a la ciudadanía como una cuestión política y social arraigada en la construcción social de la identidad nacional que surgió del colonialismo, la migración y los lazos culturales que desafían las fronteras nacionales. El artículo subraya la importancia de la ciudadanía en la democracia africana y destaca los riesgos de seguridad de las políticas de ciudadanía difusa y difícil ${ }^{4}$. Lo

4 Nota del traductor: la expresión literal utilizada por el autor es 'engorrosa'. 
más importante es que el artículo establece un vínculo crítico entre la ciudadanía y la evolución del proceso de paz marfileño. Sostiene que el hecho de no prestar suficiente atención a este problema contribuyó al colapso del acuerdo de paz diseñado por la comunidad internacional. Si bien esta era consciente del problema de la ciudadanía, como se evidencia en numerosos informes, los acuerdos de paz a menudo callaron sobre el tema ${ }^{1}$. Incluso, cuando la ciudadanía se abordó en el Acuerdo de LinasMarcoussis, se trató, en gran medida, como un problema legal y administrativo. El gran avance en el proceso de paz solo ocurrió cuando los marfileńos abordaron el tema directamente, desde una perspectiva interna, en el Acuerdo de Uagadugú.

\section{GÉNESIS DE LA GUERRA CIVIL: CIUDADANÍA Y DEMOCRACIA}

El tema de la ciudadanía es importante en muchas de las guerras civiles asociadas con las luchas por la democracia en África, como lo ilustra el trabajo de Mahmood Mamdani sobre los legados del gobierno colonial (Mamdani, 1996). Al examinar la naturaleza de los conflictos coloniales y poscoloniales en África, demuestra que las discriminaciones raciales y étnicas constituyen la negación de la ciudadanía. Esta realidad se manifiesta en la bifurcación de las personas en ciudadanos y sujetos. Mamdani examina las experiencias de las personas sometidas y las formas en que reclaman su ciudadanía a través de los movimientos de resistencia. Más recientemente, Peter Geschiere examina la ciudadanía en términos de debates políticos y sociales sobre la autoctonía; la lanza como una lucha para afirmar las raíces locales en asuntos políticos y sociales, y excluir a los extraños. Sin embargo, el extraño es difícil de definir a la luz de los patrones de migración históricos y la rápida globalización (Geschiere, 2009).

1 Hubo referencias a la ciudadanía en varios informes, entre ellos: Consejo de Seguridad de la Organización de las Naciones Unidas (ONU), "Segundo informe del Secretario General sobre la operación de las Naciones Unidas en Costa de Marfil” (S / 2004/697, onu, Nueva York, 27 de agosto de 2004); Consejo de Seguridad de las Naciones Unidas, "Tercer informe de progreso del Secretario General sobre la operación de las Naciones Unidas en Costa de Marfil" (S / 2004/962, onu, Nueva York, 9 de diciembre de 2004); "Programa de país: Côte d'Ivoire” Desarme, desmovilización y reintegración de las Naciones Unidas, (http://unddr.org/ countryprogrammes. php? c = 51) (25 de septiembre de 2009); Consejo de Paz y Seguridad, Unión Africana, "Informe del presidente de la Comisión sobre la situación en Costa de Marfil" (23. a reunión del cPs, 10-11 de enero de 2005, Libreville, Gabón). 
Ambos estudios señalan el vínculo conmovedor entre la ciudadanía y los conflictos políticos en África, y las cuestiones asociadas con la construcción social de la ciudadanía. Los problemas de ciudadanía van desde la exclusión política hasta las disputas sobre la propiedad de la tierra y las políticas de integración nacional (Dorman, Hammett y Nugent, 2007). La ciudadanía es membresía formal en un Estado basado en los principios de ius sanguinis e ius soli, y las leyes de naturalización. Asume una relación de derechos y obligaciones entre el individuo y el Estado (Marshall, 1964; Brubaker, 1992; Nyamnjoh, 2006). Aunque en principio se entienden los procedimientos para determinar la ciudadanía y se considera que los ciudadanos son iguales; en realidad estos dos requisitos fundamentales pueden ser problemáticos en estados multiétnicos con un legado de dictadura. Cuando la Unión Soviética se disolvió, por ejemplo, surgieron demandas de ciudadanía impugnadas, especialmente en los estados bálticos que tenían enormes poblaciones étnicas minoritarias rusas (Linz y Stepan, 1996). Muchos de los conflictos que rodean a la democracia en África son instigados por agravios sobre la marginación étnica, similar a la lucha por la ciudadanía en otras partes del mundo (Dorman, Hammett y Nugent, 2007). Esta desagradable realidad se manifiesta en el conflicto marfileño.

La guerra civil de Costa de Marfil fue producto de un gobierno autoritario, políticas difusas de ciudadanía e infusión de etnicidad en la democracia multipartidista (Chirot, 2006; Toungara, 2001). Después de la independencia de Francia, en 1960, el Partido Democrático de Costa de Marfil (PDCI), dirigido por Houphouët-Boigny, se convirtió en el único partido hasta 1990. Durante las elecciones multipartidistas de 1990, el PDCI derrotó al Frente de Oposición Popular Marfileña (FPI), dirigido por Laurent Gbagbo. Houphouët-Boigny es comúnmente considerado como un político habilidoso, que lidió con sus oponentes, abrió el país a la inversión extranjera y al trabajo, y cultivó fuertes (aunque subordinados) lazos con Francia (Widner, 1994; Nandjui, 1995). Sus difusas políticas de ciudadanía les permitieron tácitamente a los inmigrantes participar en la política marfileńa y desdibujaron la línea entre marfileños y no marfileños (Woods, 2003, entrevista a diplomático marfileño, Nueva York, 24 de mayo de 2005; Akokpari, 2008). Esto se convirtió en una trampa fatal para el país, ya que los políticos menos capacitados explotaron los sentimientos étnicos y nacionalistas. En Costa de Marfil, hay más de sesenta grupos étnicos clasificados en cinco grupos culturales (Akan, Krou, Norte de Mandé, Sur de 
Mandé y Gur), enredados en una fuerte división regional entre el norte dominado por los musulmanes y el sur dominado por los cristianos. La división regional se agudiza por las disparidades económicas entre el sur relativamente exitoso y el norte empobrecido. Hay enormes poblaciones de inmigrantes, especialmente de países vecinos del norte, que comparten culturas similares con los marfileños del norte (Chirot, 2006; Marshall-Fratani, 2006). En su lucha para tener éxito, HouphouëtBoigny desarrolló dos frentes: una división interna dentro del PDCI y la lucha entre el PDCI y el FPI de la oposición (Banégas, 2006). Henri Bédié se convirtió en presidente, pero no logró galvanizar el PDCI detrás de él. En 1994, un grupo disidente del PDCI, dirigido por Djéni Kobina, formó el Rassemblement des Républicains (RDR), que invitó al ex primer ministro Alassane Ouattara a ser su candidato presidencial.

El FPI también montó una vigorosa campaña contra el PDCI. Bédié hizo esfuerzos desesperados por consolidar el poder y obtener apoyo antes de las elecciones de 1995. Restringió los partidos de oposición e instigó la desagradable doctrina de la Ivoirite $^{2}$, que infundió una forma divisiva de etnicidad en la política marfileña y, sin saberlo, sembró las semillas de la guerra (Toungara, 2001; Marshall-Fratani, 2006). Estas tácticas antidemocráticas llevaron al RDR y al FPI a boicotear las elecciones presidenciales de 1995.

La doctrina de la Ivoirité tuvo un efecto escalofriante y de largo alcance en los marfileños, especialmente los norteños. La Ivoirité se remonta a los intelectuales y políticos del sur que querían definir la identidad marfileña. Desafortunadamente, la doctrina se introdujo en la política como una estratagema política nacionalistaétnica disfrazada de patriotismo (Dozon, 2000; entrevista a diplomático marfileño, Nueva York, 24 de mayo de 2005; Marshall-Fratani, 2006; entrevista a profesor de la Universidad de Cocody, Abiyán, 6 de junio de 2008).

La Ivoirité se basa en una polémica distinción entre indigenas marfileños y marfileños de ascendencia inmigrante que entrelaza sentimientos antiextranjeros y antinorteńos. El objetivo tácito de esta política étnica divisiva era marginar a los norteños, unirlos

2 Nota del traductor: Ivorité podría traducirse al español como 'marfileńalidad'; como sería de Colombia, 'colombianidad' o de España 'espańolidad'. En el artículo, se utiliza con carácter negativo y con tinte xenófobo. 
con los inmigrantes de Burkina Faso, lo que implica que ellos también son extranjeros o, en el mejor de los casos, marfileńos de ascendencia inmigrante. La Ivoirité se fue institucionalizando a través de reformas electorales y políticas de identificación nacional que inhabilitaron tácitamente a muchos marfileños del norte para buscar la presidencia y les negaron los derechos de ciudadanía. En 1994, Bédié impulsó, a través de la Asamblea Nacional dominada por el PDCI, una ley que requería que los candidatos a la presidencia y la legislatura demostraran que ellos y sus padres fueran marfileños por origen (Toungara, 2001; Marshall-Fratani, 2006; Banégas, 2006; Geschiere, 2009). Esta ley se incorporó a la Constitución de 2000. Según el artículo 35, un candidato a la presidencia "debe ser marfileńo de nacimiento, nacido de un padre y de una madre marfileña por nacimiento. Nunca debió renunciar a la nacionalidad marfileña. Nunca debe haber tenido otra nacionalidad. Debe haber residido en Costa de Marfil continuamente durante los cinco años anteriores a la fecha de las elecciones y haber totalizado diez años de presencia efectiva" (Constitución de Costa de Marfil). Las leyes fueron diseñadas para descalificar al líder político del norte de Ouattara, cuyo padre supuestamente es de Burkina Faso ${ }^{3}$.

La Ivoiritétambién se filtró a otras áreas de la sociedad marfileña, como la identificación nacional, la tenencia de la tierra y las políticas de empleo del sector público. El Gobierno se negó a emitir certificados de nacionalidad a muchas personas del norte, alegando que no eran marfileńos porque no tenían los documentos adecuados para demostrar que sus padres eran marfileños. Según las políticas de identificación adoptadas por el gobierno de la FPI en 2001, por ejemplo, "cualquier persona que solicite un documento de identidad debe demostrar su nacionalidad mediante la obtención de una declaración de origen emitida por un comité de su aldea de origen" (Banégas, 2006, p. 542).

Esencialmente, la nacionalidad marfileña se basaba en la capacidad de demostrar el estado de acólito de los autóctonos de la aldea marfileńa. Se adoptaron las controvertidas políticas de nacionalidad, a pesar de que estaba claro que las brechas

3 Ouattara vivió fuera del país durante muchos ańos como estudiante y funcionario del Fondo Monetario Internacional y del Banco Central de los Estados de África Occidental. Se cree que tenía un pasaporte de Burkina Faso (véase Toungara, 2001). 
en los registros de nacimiento harían difícil proporcionar pruebas documentales de ascendencia. Además, el legado de las políticas de ciudadanía colonial francesa, la definición poco clara de ciudadanía de Houphouët-Boigny, la fluidez cultural de las regiones fronterizas, la larga historia de migración interna y urbanización, y la gran cantidad de inmigrantes arraigados de los países vecinos, hicieron que las políticas fueran poco realistas. Como resultado de la Ivoirité, los norteños no solo se sintieron marginados políticamente, sino que también se vieron a sí mismos como víctimas de discriminación patrocinada por el Estado en la aplicación de las leyes de ciudadanía. Esta sensación de negación injusta de la ciudadanía se convirtió en la base de las protestas políticas y la guerra civil. Desde sus inicios, la Ivoirité se convirtió en el problema político central en Costa de Marfil.

La crisis fomentada por Bédié se vio exacerbada por el golpe de Estado del 25 de diciembre de 1999, que llevó al poder al general Robert Guéï (Banégas y Losch, 2002). La resistencia popular al gobierno militar coincidió con la lucha de poder tripartita y una vehemente campaña dirigida por el RDR para terminar con la Ivoirité. En un intento por manipular las elecciones presidenciales de octubre de 2000, Guéï suprimió la libertad política y utilizó la doctrina de la Ivoirité para descalificar a Ouattara. En protesta, el PDCI y el RDR boicotearon las elecciones presidenciales. Guéï detuvo el conteo de votos, disolvió la comisión electoral y se declaró arbitrariamente ganador. Las masivas protestas callejeras de los partidarios del FPI obligaron a Guéi a huir, allanando el camino para que Gbagbo se instalara como presidente (British Broadcasting Corporation [BвC], 2000a; 2000b; 2000c; Akokpari, 2008). Estalló una nueva violencia entre el FPI y el RDR; y este último exigió una nueva votación, basada en políticas de ciudadanía inclusivas que no privarían de derechos a los norteños. Gbagbo insistió en su mandato electoral, mientras que la RDR y los descontentos del norte rechazaron su gobierno, calificándolo como ilegítimo. Gbagbo también continuó apoyando la Ivoirité, mientras insinuaba que Ouattara no era un marfileño indígena $\mathrm{y}$, como tal, no estaba calificado para ser presidente (Banégas, 2006; Akokpari, 2008).

La debacle política degeneró en una guerra civil después del golpe de Estado del 19 de septiembre de 2002, provocada por informes de un inminente programa de desmovilización involuntaria, que se cree que es parte de la purga de la Ivoirité 
contra los norteños. Las fuerzas rebeldes del Movimiento Patriótico de Costa de Marfil (мРCI) atacaron Abiyán y ciudades del norte (ввС, 2002). Los rebeldes se retiraron a sus bases en el norte después de que las fuerzas leales rechazaran el ataque de Abiyán. Los objetivos declarados del MPCI eran derrocar al gobierno de Gbagbo, celebrar elecciones inclusivas y reinstalar a todos los soldados disueltos. A finales del 2002, habían surgido dos grupos rebeldes más pequeños. Tanto el Movimiento por la Justicia y la Paz (MJP) como el Movimiento Popular Marfileńo del Gran Oeste (MPIGo) expresaron intenciones similares para derrocar a Gbagbo (ввС, 2003). Los rebeldes se reagruparon en un nuevo movimiento llamado Forces Nouvelles (FN) bajo el liderazgo de Guillaume Soro. El FN también incluye dozos (cazadores tradicionales) y soldados disidentes que apoyan al general Guéi. A finales del 2002, la crisis política de Costa de Marfil había degenerado en una guerra civil. El Gobierno había perdido el control del norte, mientras la hostilidad continuaba creciendo entre norteños y sureños. El país también se vio empañado por luchas comunitarias y manifestaciones políticas violentas, que enfrentaron a los Jóvenes Patriotas (YP) pro-FPI contra los partidarios de la oposición (Woods, 2003; Marshall-Fratani, 2006). A finales del 2003, más de 700000 personas habían sido desplazadas; y un número indeterminado, asesinadas (International Crisis Group, 2003).

Como la mayoría de los conflictos en África, las verdaderas disputas étnicas en Costa de Marfil a menudo quedaron fuera de las declaraciones oficiales, y la lucha para terminar con la Ivoirité fue enmascarada como una lucha por la democracia. Al tratar de expulsar a Gbagbo y celebrar elecciones inclusivas, los rebeldes estaban luchando para detener la marginación de los norteños. Como el mismo Soro confesó, la rebelión fue instigada por la insatisfacción con las elecciones del 2000 y por la Ivoirité, que calificó como el concepto xenófobo de "Costa de Marfil para los marfileños"; es decir que, para aquellos que viven en el Sur, los norteños son considerados extranjeros en su propio país. Los rebeldes querían asegurarse de que los norteños recibieran sus documentos de ciudadanía y se les permitiera una participación igualitaria en la política. La democracia para ellos significaba un reconocimiento inequívoco de su ciudadanía e igualdad de derechos como marfileńos. Son precisamente estos agravios en relación con la ciudadanía los que formaron el nexo crítico entre la democracia y la guerra civil, y que no recibieron la atención adecuada en los acuerdos de paz mediados internacionalmente. 


\section{MEDIACIÓN INTERNACIONAL DE LA PAZ: DESCUIDO DE LA CIUDADANÍA}

La mediación internacional por la paz en Costa de Marfil fue encabezada por la Comunidad Económica de los Estados de África Occidental (Cedeao), las Organización de las Naciones Unidas (ONU), la Unión Africana (UA) y Francia. La Cedeao y la onU vieron el conflicto como un grave problema de seguridad regional, especialmente debido a las especulaciones sobre la participación extranjera, la frágil seguridad en los países vecinos y la centralidad económica de Costa de Marfil. Francia, que tiene profundos intereses económicos y de seguridad en Costa de Marfil, también trató la crisis con urgencia. Países africanos como Togo, Mali, Angola, Nigeria, Sudáfrica, Ghana, Senegal y Burkina Faso se involucraron en el esfuerzo de mediación de paz. La comunidad internacional desplegó rápidamente fuerzas de paz y convocó conversaciones de paz.

Aunque los acuerdos de paz mediados internacionalmente no resolvieron el conflicto, la intervención internacional contuvo el choque armado y mejoró la situación humanitaria. Creó oportunidades para el diálogo y sentó las bases para el Acuerdo de Uagadugú. Los ingredientes típicos de los acuerdos de paz mediados internacionalmente en los conflictos africanos son las disposiciones sobre el alto el fuego, el poder compartido, el desarme, los derechos humanos y las elecciones (Mehler, 2009). El camino hacia la paz se basa en elecciones democráticas exitosas (Goulding, 1999; entrevista a oficial de la onU, Nueva York, 24 de mayo de 2005; entrevista a diplomático europeo, Abiyán, 6 de junio de 2008). En Costa de Marfil esta receta falló porque no prestó suficiente atención a la ciudadanía, que es la causa subyacente de la guerra. Con la excepción del Acuerdo de Linas-Marcoussis, los acuerdos de ingeniería internacional callaron frente al problema de la ciudadanía; en cambio, se centraron en la distribución del poder, el desarme y las elecciones. Los resultados finales fueron sucesivos acuerdos de paz fallidos.

Cuando estalló la lucha, la Cedeao y Francia adoptaron una postura proactiva para poner fin al conflicto. Las conversaciones de paz se llevaron a cabo apresuradamente en Accra, Lomé y París. Durante la Conferencia de París en enero del 2003, los beligerantes firmaron el Acuerdo de Linas-Marcoussis — los partidos del acuerdo son 
los principales partidos políticos (PDCI, RDR, FPI), grupos rebeldes (MPCI, MJP, MPIGO) y partidos políticos más pequeños (Mouvement des Forces del'Avenir, Parti Ivoirien des Travailleurs, Union Democratique et Citoyenne, y Unión para la Democracia y la Paz en Costa de Marfil) - . El yp inmediatamente realizó una manifestación contra el acuerdo, que vio como una estratagema francesa contra el Gobierno. Irónicamente, este acuerdo imperfecto se convirtió en la piedra angular de los acuerdos posteriores negociados por la comunidad internacional. Como era de esperarse, Gbagbo demostró repetidamente su disgusto por las soluciones internacionales al conflicto (ввС, 2003a; 2003b; 2003c; entrevista a diplomático marfileño, Nueva York, 24 de mayo de 2005; entrevista a diplomático africano, Abiyán, 7 de junio de 2008).

El Acuerdo de Linas-Marcoussis identificó los problemas del conflicto, pero su fórmula para la paz fracasó. La parte principal del acuerdo se centró en la distribución del poder, en lugar del tema central de ciudadanía. Sustancialmente, se ocupó solo de la formación de un Gobierno de Reconciliación Nacional (GNR), compuesto por representantes de cada uno de los signatarios del acuerdo y un primer ministro consensuado. La misión crítica del GNR era reestructurar las fuerzas de seguridad y organizar elecciones creíbles. El anexo del acuerdo detallaba la agenda del GNR, que incluía ciudadanía, elecciones, tenencia de la tierra, medios de comunicación, derechos humanos y reformas económicas, más un programa de desarme. También pidió un alto el fuego, una amnistía y la restauración de la autoridad estatal.

La cuestión de la ciudadanía no solo se relegó al anexo del acuerdo, sino que también se trató de manera simplista y potencialmente peligrosa. El Acuerdo de Linas-Marcoussis reconoció las leyes de ciudadanía marfileña de 1961 y 1972 como generosas y bien redactadas, pero señaló problemas en su aplicación que condujeron a la negación injusta de los derechos de ciudadanía. Se le pidió al Gobierno que simplificara la aplicación de las leyes, suspendiera su programa de identificación y estableciera una Comisión Nacional de Identificación para supervisar un nuevo sistema de identificación. Para aliviar el problema de identificación, el acuerdo exigía la eliminación del requisito de permiso de residencia para los ciudadanos de la Cedeao. El acuerdo se refería a la ciudadanía, pero lo trataba como un problema administrativo que podría rectificarse mediante la aplicación adecuada de las leyes vigentes. No logró abordar la construcción social de la ciudadanía y proporcionar una solución política al problema. 
El acuerdo se derrumbó bajo el peso de una comprensión conflictiva de la ciudadanía. El gobierno de Gbagbo insistió en una definición legal limitada de ciudadanía, registros de nacimiento oficiales y lazos ancestrales verificables con las aldeas marfileñas. Los norteños desafiaron el espíritu de la interpretación de las leyes. Lo vieron como insensible a las brechas en los registros de nacimiento, las raíces urbanas de muchos marfileños y las dislocaciones sociales que emanan de la migración interna.

Los sureños continuaron viendo la ciudadanía en términos de ascendencia profundamente arraigada que se remonta, al menos, al período colonial temprano, principalmente en la línea de ius sanguinis. Además, hicieron una distinción entre los privilegios políticos de los ciudadanos que tenían ascendencia profundamente arraigada en Costa de Marfil (es decir, los marfileńos indígenas) y aquellos cuyos antepasados se establecieron en Costa de Marfil durante el periodo colonial tardío y subsiguiente (marfileños de ascendencia inmigrante).

Los norteños vincularon la ciudadanía al lugar de nacimiento (iussoli) y al asentamientoque no necesariamente se remonta a la era precolonial— $\mathrm{y}$ atacaron la indigenidad como una construcción legal o política poco realista en un país creado a partir del colonialismo francés (entrevista a hombre de negocios y líder comunitario del norte, Abiyán, 7 de junio de 2008). Para ellos, las distinciones entre supuestos indígenas marfileños y marfileños de ascendencia inmigrante era una forma de discriminación étnica patrocinada por el Estado, que infringía su ciudadanía y los agrupaba erróneamente con inmigrantes de países vecinos del norte simplemente por compartir las mismas culturas (MarshallFratani, 2006; Banégas, 2006; Akokpari, 2008 y Geschiere, 2009).

Con respecto a la elegibilidad parala presidencia, el Acuerdo de Linas-Marcoussis cuestionó el lenguaje de la Constitución del 2000. El acuerdo recomendó que un candidato "debe tener solo la ciudadanía marfileńa y tener un padre o una madre nacidos en Costa de Marfil”, en lugar del lenguaje del artículo 35 de la Constitución del 2000, que requiere que un candidato "debe ser marfileńo de nacimiento, nacido de un padre y de una madre marfileña por nacimiento". El acuerdo exigía una aclaración de las condiciones bajo las cuales la ciudadanía marfileña podría ser revocada, para evitar la ambigüedad en la elegibilidad de la presidencia. Si bien esto tenía la intención de conciliar las dos 
nociones divergentes de ciudadanía, el acuerdo redujo inadvertidamente el problema de la ciudadanía a una mera lucha por el poder. No pudo abordar las disputas sobre la definición y la prueba de ciudadanía. Las partes beligerantes se escudaron en sus propias definiciones de ciudadanía y se disputaron los registros de nacimiento y los certificados de nacionalidad, lo que envenenó todo el acuerdo.

La formación del GNR se estancó a pesar del nombramiento de Seydou Diarra como primer ministro. Los desacuerdos sobre la asignación de puestos ministeriales y los poderes del primer ministro llevaron a un punto muerto el proceso de paz (ввс, 2003a). En marzo del 2003, el grupo de contacto de la Cedeao sobre Costa de Marfil negoció el Acuerdo Accra II. El acuerdo no solo reafirmó el de LinasMarcoussis, sino que continuó tratando el conflicto como una mera lucha por el poder. La autoridad de Gbagbo como jefe de Estado, comandante en jefe y garante de la constitución y la República se reafirmó como un gesto para difuminar el tema de la legitimidad en relación con su controvertida elección. Recíprocamente, se acordó aplicar los términos del Acuerdo de Linas-Marcoussis, especialmente facilitando la formación del GNR y delegando el poder al primer ministro. Las partes enfrentadas acordaron crear un Consejo de Seguridad Nacional de quince miembros, compuesto por el presidente, el primer ministro y un representante de cada uno de los signatarios del acuerdo, los militares, la gendarmería y la policía. El Consejo debía supervisar los ministerios de defensa y el interior.

Tras el Acuerdo de Accra II, se mantuvieron conversaciones directas entre las Fuerzas Armadas Nacionales de Costa de Marfil (Fanci) y las Fuerzas Armadas de las Fuerzas Nouvelles (FAFN) y las fuerzas de paz desplegadas por la ONU. El acuerdo se estancó mientras continuaban los enfrentamientos violentos entre la oposición y los partidarios del Gobierno, mientras los rebeldes controlaban el norte. La Cedeao, la UA y la ONU ahora encabezaron una nueva iniciativa de paz. Las partes enfrentadas y líderes de dieciséis estados africanos (incluidos doce jefes de estado) se reunieron en Accra a finales de julio del 2004 y firmaron el Acuerdo de Accra III. La reunión buscó ejercer una inmensa presión regional sobre las facciones marfileñas para implementar los acuerdos anteriores. Desafortunadamente, no logró abrir un nuevo camino de paz. En cambio, se empantanó con referencias persistentes al extinto Acuerdo de Linas-Marcoussis. Se instó a Gbagbo a implementar las disposiciones de Linas-Marcoussis sobre elegibilidad 
para la presidencia a finales de septiembre de 2004. A su vez, todas las facciones se comprometieron a apoyar la adopción de las reformas legales previstas en el acuerdo para fines de agosto de 2004. Gbagbo acordó emitir un decreto que especificara los poderes del primer ministro con base lo acordado, y las partes enfrentadas se comprometieron a comenzar el desarme antes del 15 de octubre del 2004, de conformidad con los términos del Acuerdo de Linas-Marcoussis y los acuerdos de Grand Bassam y Yamoussoukro. Se solicitó al GNR que estableciera un cronograma para la restauración de la administración estatal y los servicios públicos en todo el país.

Después del Acuerdo de Accra III, la Asamblea Nacional hizo esfuerzos para aprobar las reformas previstas en Linas-Marcoussis. Sin embargo, la situación militar se deterioró cuando las fuerzas gubernamentales bombardearon posiciones rebeldes y golpearan bases militares francesas en noviembre del 2004. Las represalias francesas contra las fuerzas gubernamentales provocaron violentas protestas antifrancesas, lo que empeoró la precaria situación (вBC, 2004a).

El Acuerdo de Pretoria de abril del 2005, mediado por el presidente Thabo Mbeki, en nombre de la UA, reunió a Gbabgo, Soro, Bédié, Ouattara y Diarra. Se centró en los asuntos militares y electorales urgentes. La pieza central del componente militar fue el desarme y el desmantelamiento de las milicias armadas. Los jefes de personal de las Fanci y las fafN debían implementar el plan nacional de desarme, desmovilización y reintegración de inmediato, y hacer recomendaciones específicas para integrar a los ejércitos y reestructurar las fuerzas de seguridad conforme con el Acuerdo de Linas-Marcoussis.

El acuerdo subrayaba la necesidad de celebrar elecciones presidenciales en octubre del 2005, que serían seguidas por elecciones legislativas. La composición de la Comisión Electoral Independiente (IEC) fue modificada. Cada uno de los signatarios del Acuerdo de Linas-Marcoussis tendría dos representantes en la Comisión Central, en el entendimiento de que seis serían de las FN. La Mesa de la Comisión Central estaría compuesta por un representante del presidente, el presidente de la Asamblea Nacional y cada una de las diez partes en el Acuerdo de Linas-Marcoussis. Pero el predecible incumplimiento del acuerdo fue evidente 
en la falta de consenso sobre la elegibilidad para la presidencia. Aunque más tarde, Gbagbo anunció que cualquier candidato nominado por las partes del acuerdo sería elegible para participar en las elecciones presidenciales, el problema de ciudadanía subyacente aún no se había resuelto.

\section{CONVERSACIONES DIRECTAS: CAMINO DE CIUDADANÍA A LA PAZ}

El Acuerdo de Pretoria languideció a medida que la violencia continuó, el desarme se estancó, las elecciones se volvieron esquivas y el Gobierno siguió siendo disfuncional (International Crisis Group, 2005; ввC, 2006). En diciembre del 2005, el primer ministro Diarra fue reemplazado por otro tecnócrata muy respetado, Charles Banny, quien también se enfrentó a obstáculos innumerables en la implementación de los acuerdos de paz. Los líderes de Costa de Marfil se estaban cansando de los acuerdos negociados internacionalmente a medida que las opciones de mediación disminuían. El Acuerdo de Uagadugú se formó dentro de este atolladero.

Desde su firma, en marzo de 2007, por el gobierno de FPI y las FN, la seguridad había mejorado enormemente y se vislumbraba una paz duradera, a pesar de los retrasos en la implementación (un Security Council, 2009). El acuerdo fue precedido por dos acontecimientos clave que alteraron el cálculo político en Costa de Marfil. Primero, había una clara sensación de fatiga de guerra entre el pueblo y la comprensión de la élite de que la victoria militar absoluta era esquiva (entrevista a consejero de presidencia, Abiyán, 5 de junio de 2008; entrevista a diplomático africano, Abiyán, 7 de junio de 2008). Esta realidad hizo eco en comentarios conciliadores de Gbagbo y Soro. En su discurso del 19 de diciembre del 2006 a la nación, Gbagbo hizo un llamado para el diálogo directo con los rebeldes. Soro respondió positivamente en su discurso de Año Nuevo (Colombant, 2006; un Security Council, 2007).

El otro acontecimiento fue la adopción de la Resolución 1721 del Consejo de Seguridad de la ONU, que expandió significativamente el poder del primer ministro. Gbagbo, cuya legitimidad se basó, en gran medida, en las extensiones respaldadas por la ONU de su mandato electoral vencido, vio la Resolución 1721 como una seria 
amenaza para su presidencia. Además de la dinámica interna, hubo cambios en la actitud del presidente Blaise Compaore de Burkina Faso, sospechoso de apoyar a los rebeldes (Banégas y Losch, 2002).

Compaore entendió que la paz en Costa de Marfil aseguraría el flujo de remesas a Burkina Faso y repararía su imagen empañada como partidaria de los señores de la guerra. La combinación de sensación de vulnerabilidad, fatiga de guerra, falta de un camino claro hacia la victoria militar y la posición cambiante de Burkina Faso, proporcionaron un terreno fértil para el compromiso. El Acuerdo de Uagadugú se basó en la confianza mutua asumida entre los beligerantes y el facilitador, un espíritu de diálogo y paridad entre el Gobierno y las FN (entrevista a consejero de la presidencia, Abiyán, 5 de junio de 2008). Este entendimiento tácito entre Gbagbo y Soro se reforzó en las disposiciones del código de conducta y las generosas amnistías que cubrían los posibles delitos relacionados con la seguridad nacional cometidos desde el 17 de septiembre de 2000. El Acuerdo de Uagadugú se apartó de la fórmula de paz establecida en el Acuerdo de Linas-Marcoussis y marcó un cambio monumental en el proceso de paz.

A diferencia de todos los demás acuerdos, el de Uagadugú fue encabezado por marfileños. Como se señaló en el acuerdo, Gbagbo le pidió personalmente al presidente Compaore, en enero del 2007, que facilitara las conversaciones directas entre su gobierno y las FN. Lo más notable es que el acuerdo abordó directamente el problema de ciudadanía. Desde el principio, los beligerantes reconocieron que "la identificación de las poblaciones marfileñas y extranjeras que viven en Costa de Marfil es una gran preocupación. La ausencia de un documento de identidad claro y estándar y de documentos administrativos individuales que certifiquen la identidad y la nacionalidad de las personas es una fuente de conflicto" (Acuerdo de Ouagadougou, artículo 1). Al reconocer este hecho, los líderes marfileños demostraron un profundo entendimiento y comprensión de la causa de la guerra.

Para resolver el problema de la ciudadanía, los protagonistas acordaron proporcionar credenciales a todos los marfileños que no tenían los documentos adecuados y establecer un sistema de identificación confiable. Según el acuerdo, los tribunales 
móviles (audiences foraines), presididos por un juez, emitirían certificados de nacimiento sustitutos (jugements completifs) a "individuos nacidos en Costa de Marfil que nunca se han registrado en una oficina de registro" (Acuerdo de Ouagadougou, artículo 1.1.2). Estas personas irían a una audiencia en la corte móvil correspondiente a su lugar de nacimiento durante un período de gracia de tres meses. Los registros de nacimientos que se perdieron o destruyeron en las oficinas de registro debían ser reconstituidos. Se debían emitir nuevos documentos de identidad a prueba de falsificaciones a marfileños y extranjeros.

Los extranjeros, que tenían un certificado de nacimiento o un certificado de nacimiento sustituto, y un documento de su consulado que acreditara su nacionalidad, recibirían permisos de residencia. Los marfileños recibirían sus nuevas tarjetas de identidad nacionales a través del proceso de identificación estándar o "identificación basada en el nuevo padrón electoral” (Acuerdo de Ouagadougou, artículo 1.3).

Según la identificación estándar, los marfileños que tenían un certificado de nacionalidad y un certificado de nacimiento o un certificado de nacimiento sustituto, recibirían la nueva tarjeta de identidad nacional. Todos los demás recibirían sus tarjetas de identidad nacionales después de registrarse en el censo electoral. Los marfileños que habían cumplido los dieciocho años y tenían un certificado de nacimiento o un certificado de nacimiento sustituto, tenían derecho a registrarse en el censo electoral. Las tarjetas de identidad nacionales se emitirían después de que se fuera validado el censo electoral. La IEC, con la ayuda del Instituto Nacional de Estadística y una agencia técnica contratada, fue responsable de crear un censo electoral preciso ${ }^{4}$. El acuerdo preveía el uso de datos biométricos para asegurar una identificación precisa.

Aunque el acuerdo no definió la ciudadanía marfileña, proporcionó un mecanismo para resolver los problemas de ciudadanía asociados con la Ivoirité. Los artículos 6 y 7 de la Ley de Nacionalidad existente definen a un ciudadano marfileńo como cualquier persona nacida de al menos uno de los padres marfileños o alguien nacido

4 La Société d'Application Générales Electriques et Mécaniques era esta agencia técnica. 
en Costa de Marfil de padres desconocidos (República de Costa de Marfil, s. f.). Existen disposiciones para la adquisición de la ciudadanía por adopción, matrimonio, naturalización o reintegración. El conflicto anterior sobre la Ivoirité se centró en el intento de definir estrictamente la elegibilidad para la presidencia sobre la base de la ascendencia y la documentación del lugar de nacimiento, y la ascendencia para determinar la ciudadanía marfileña.

El Gobierno insinuó que muchos norteños reclamaban falsamente la ciudadanía marfileña, mientras que los norteños se quejaron de que este se negaba maliciosamente a emitir certificados de nacionalidad a ciudadanos marfileńos nativos del norte. Como observó Daniel Chirot, los auténticos marfileños del norte se encontraron en una situación precaria. Muchos no tenían documentos completos, debido a registros faltantes, reubicación familiar o confiscación de sus documentos por agentes de seguridad (Chirot, 2006; Marshall-Fratani, 2006). Algunos de los líderes del sur entrevistados insistieron en aplicar las leyes y poner la carga de la prueba de ascendencia marfileña en los individuos. En su opinión, todos los marfileños genuinos podrían obtener los registros necesarios para demostrar su ascendencia marfileña. Sin embargo, destacaron que los registros no son perfectos (entrevista a abogado y líder comunitario, Abiyán, 11 de junio de 2008). Además, entendieron que la urbanización había debilitado los lazos con las aldeas ancestrales.

Los problemas de la Ivoirité se resumieron en la lucha de Ouattara con los sucesivos gobiernos posteriores a Houphouët-Boigny. Bédié afirmó que el padre de Ouattara era de Burkina Faso y, por implicación, no era un marfileño genuino. Ouattara insiste en que sus padres nacieron en Costa de Marfil. Cuando el juez Zoro Ballo le otorgó a Ouattara un certificado de nacionalidad en septiembre de 1999, el Gobierno investigó al juez y lo obligó a renunciar. El gobierno de Bédié acusó a Ouattara de falsificar sus documentos y revocó el certificado en octubre de 1999 (Langer, 2008). El relato de Ouattara ejemplificó la discriminación y la impracticabilidad de las políticas de ciudadanía de la Ivoirité. Si bien los criterios restrictivos para la presidencia no afectaron a la gran mayoría de los norteños, la negativa a emitirles certificados de nacionalidad era una preocupación seria para esta población en general. 
El Acuerdo de Uagadugú proporcionó un mecanismo para resolver el conflicto sobre la ciudadanía que era razonable y consistente con la ley marfileña. Los tribunales móviles aceptaron documentos y testimonios en sus deliberaciones. Los peticionarios que no tenían evidencia documentada podrían traer a dos testigos para dar fe de su lugar de nacimiento o la ciudadanía de al menos uno de sus padres (República de Costa de Marfil, 2007). Los peticionarios ya no necesitaban ir a su aldea ancestral. En cambio, podrían presentar una solicitud en su lugar de nacimiento. Este mecanismo fue diseñado para llenar vacíos en los registros y reconocer la dislocación social resultante de la migración interna. Proporcionó un camino realista para que los marfileños probaran su lugar de nacimiento y la ciudadanía de sus padres y así establecieran su legítimo reclamo de ciudadanía marfileña.

Este mecanismo de identificación, junto con la disposición que extiende la ciudadanía a las personas nacidas en Costa de Marfil a padres desconocidos, debería abordar drásticamente la distinción problemática entre los marfileños indígenas y los marfileños de ascendencia inmigrante. Las únicas categorías restantes de ciudadanía serían: marfileños por nacimiento basados en ius sanguinis y marfileños por adquisición basada en adopción, matrimonio o naturalización. Los norteños tendrían los documentos para demostrar que son marfileños por nacimiento, al igual que los sureños.

El Acuerdo de Uagadugú abordó las cuestiones militares que paralizaron los acuerdos anteriores y proporcionó un mecanismo para el diálogo continuo. Las partes beligerantes acordaron reestructurar las dos fuerzas armadas y establecer una Estructura de Comando Integrado bajo el mando conjunto del jefe de personal de las Fanci y el jefe de personal de las FAFN. El comando integrado fue el responsable de desarmar y reintegrar a los combatientes, garantizar la libre circulación de personas y bienes, y proporcionar seguridad. Acordaron eliminar las zonas de confianza dirigidas por el personal de mantenimiento de la paz francés y de la onU, facilitar la libre circulación en todo el país y volver a desplegar la administración en el norte. El acuerdo creó dos órganos de alto nivel, el Marco de Consulta Permanente (PCF) y el Comité de Evaluación y Monitoreo (EMC), para facilitar la continuación de las conversaciones directas. El PCF, compuesto por Gbabgo, Soro, Ouattara, Bédié y Compaore, es el órgano de supervisión y diálogo permanente. El EMC, que supervisa la implementación del acuerdo, está compuesto por el facilitador, tres representantes 
del Gobierno y tres de las FN. Los dos partidos podrían extender conjuntamente la membresía a otras fuerzas políticas marfileñas. El facilitador también podría invitar a observadores y miembros de la comunidad internacional. Este marco para el diálogo continuo ha demostrado ser invaluable para ajustar el cronograma de implementación y mantener el acuerdo en camino.

Un elemento crítico del acuerdo de paz, no mencionado en el texto del Acuerdo de Uagadugú, fue el acuerdo de reparto de poder entre Gbagbo y Soro. Gbagbo nominó a Soro para ser primer ministro poco después de firmar el acuerdo. Este arreglo no solo satisfizo los egos políticos de los dos líderes, sino que también les dio una influencia considerable sobre el futuro político del país. Gbagbo salvó su presidencia y ganó una plataforma sólida desde la cual lanzar otra candidatura presidencial. Como poderoso primer ministro, Soro tiene una posición sólida para dar forma a la implementación del acuerdo y entregar a los norteños sus documentos de ciudadanía. Para Soro, esto podría ser una reivindicación de la rebelión y un camino para convertirse en un héroe en el norte.

\section{CONCLUSIÓN: DESAFÍOS POSTERIORES A UAGADUGÚ Y CONSOLIDACIÓN DE LA PAZ}

El Acuerdo de Uagadugú es un hito en el conflicto, porque identificó claramente el problema clave de la ciudadanía y describió un mecanismo para rectificarlo. La consolidación de la paz, sin embargo, se basa en su implementación. Se ha logrado un progreso significativo, la lucha armada se ha detenido, el Gobierno se ha mantenido en gran medida estable, ha comenzado el desarme, se han emitido certificados de nacimiento sustitutos a un número significativo de personas y el proceso de identificación y registro de votantes está progresando (UN Security Council, 2009). No obstante, estos procesos han sido demasiado lentos e incompletos. A pesar de su compromiso con el acuerdo, la implementación se ha visto obstaculizada por problemas logísticos y cálculos estratégicos por parte de los beligerantes, para no ser burlados durante el proceso de implementación. 
Para marzo del 2008, los tribunales móviles habían emitido 372810 certificados de nacimiento sustitutos (Oficina del Primer Ministro, República de Costa de Marfil, 2008). Las audiencias, que habían estado ocurriendo de manera intermitente, se relanzaron en agosto del 2008 para que se pudiera cubrir a más personas. El registro de votantes comenzó en septiembre del 2008. A finales de año, había alrededor de 3000 sitios de registro y aproximadamente 2.8 millones de personas se habían registrado (un Security Council, 2009).

El 14 de mayo del 2009, Soro anunció que las elecciones se celebrarían el 29 de noviembre del 2009. Más de seis millones de votantes se registraron para este período electoral, que nuevamente se pospuso (Gobierno de Costa de Marfil, 2009). En el lado militar, el desarme progresó, aunque lentamente. A finales del 2008, 11769 de los 34678 combatientes las FN provistos habían pasado por el ejercicio de acantonamiento (UN Security Council, 2009). Algunos combatientes las FN se integraron en los servicios de seguridad, pero el desacuerdo sobre los salarios y las largas esperas han retrasado el proceso.

A pesar de la paz, las ganancias del Acuerdo de Uagadugú se vieron socavadas por la imposibilidad de celebrar elecciones. En febrero de 2010, Gbagbo disolvió el Gobierno y la IEC, a los que acusó de fraude en el registro de votantes. La oposición acusó a Gbagbo de tratar de retrasar las elecciones y aferrarse al poder. Aunque el primer ministro Soro formó un nuevo gobierno, no hay una fecha clara para las elecciones. Ahora existe un peligro real de que el acercamiento entre el gobierno de las FN y el FPI se derrumbe o que el statu quo conduzca a una nueva generación de dictaduras.

La lección fundamental para los responsables políticos y académicos es cómo abordar los conflictos que surgen en las transiciones democráticas. Claramente, el reconocimiento temprano de la causa principal de un conflicto y un enfoque persistente en el tema proporcionaría mejores oportunidades para la paz. El Acuerdo de Uagadugú debería ser una lección para el establecimiento de la paz en África. Como sostengo, el fracaso del acuerdo de paz mediado internacionalmente 
se debe, en parte, al descuido del problema de ciudadanía subyacente. Los mediadores internacionales estaban al tanto de la disputa sobre la ciudadanía, pero a los acuerdos no les prestaron la debida atención. Esta aparente negligencia es desconcertante y plantea preguntas sobre la cultura y las instituciones de la mediación para la paz.

Las mediaciones internacionales en los conflictos africanos tienden a centrarse, en gran medida, en el cese el fuego, el poder compartido, las elecciones y los asuntos humanitarios. Esta fue la receta para la paz empleada por la onU y la Cedeao en Liberia y Sierra Leona. Tuvo éxito porque las guerras civiles fueron, en su mayoría, luchas por el poder. Parece que la mediación internacional en Costa de Marfil estaba demasiado unida a la fórmula tradicional de paz empleada en los países vecinos. Tanto la ONU como la Cedeao adoptaron un enfoque regional de los conflictos en África Occidental. En el proceso, pasaron por alto las diferencias críticas en las causas subyacentes de las guerras civiles en Costa de Marfil y en Liberia y Sierra Leona.

Costa de Marfil muestra que el cese de hostilidades, el poder compartido y las elecciones solo funcionan si los acuerdos de paz resuelven los agravios subyacentes. La guerra civil de Costa de Marfil demuestra las conexiones implícitas entre democracia, etnia y ciudadanía. Esta realidad subraya la necesidad de reconocer los significados matizados de la democracia, que a menudo se invoca como una tapadera para la liberación étnica. Con frecuencia, la democracia se reduce a elecciones multipartidistas liberales, lo que supone que los individuos son ciudadanos libres de lazos étnicos. En Costa de Marfil la transición democrática expuso los sentimientos étnicos políticamente envenenados y la noción subdesarrollada de ciudadanía. Los marfileños están aprendiendo dolorosamente que la democracia requiere un Estado nación, que debe definirse en términos no solo de territorio, sino también de membresía. Este tema desatendido y descuidado de la ciudadanía convirtió la transición democrática marfileńa en una guerra civil e hizo difícil la paz durante mucho tiempo. 


\section{Referencias}

Adebajo, A. (2001). Building Peace. En J. Hirsch (ed.), Sierra Leone: Diamonds and the struggle for democracy. Boulder, co: Lynne Rienner Publishers.

Adebajo, A. (2002). Building Peace in West Africa: Liberia, Sierra Leone, and Guinea-Bissau. Boulder, co: Lynne Rienner Publishers.

Adebajo, A. y Rashid, I. (eds.). (2004). West Africa's Security Challenges: Building Peace in a Troubled Region. Boulder, co: Lynne Rienner Publishers.

Adeleke, A. (1995). The Politics and Diplomacy of Peacekeeping in West Africa: The EcowAs Operation in Liberia. Journal of Modern African Studies, 33(4), 569-597.

Akokpari, J. (2008). "You don't belong here": Citizenship, the State and Africa's Conflicts: Reflections on Ivory Coast. En A. Nhema y T. Zeleza (eds.), The Roots of African Conflicts: The Causes and Costs (pp. 88-105). Oxford: James Currey.

Banégas, R. y Losch, B. (2002). La Côte d'Ivoire au bord de l'implosion. Politique Africaine, 87, 139-161.

Banégas, R. (2006). Côte d'Ivoire: patriotism, ethnonationalism and other African modes of self-writing. African Affairs, 105(421), 535-552.

British Broadcasting Corporation (BBC). (2000a). Clashes at Ivory Coast army camp, $B B C$ News. Recuperado de http://news.bbc.co.uk/2/hi/africa/ 988935.stm

British Broadcasting Corporation (BBC). (2000b). Ivory Coast bars opposition leader, $B B C$ News. Recuperado de http://news.bbc.co.uk/2/hi/africa/960415.stm

British Broadcasting Corporation (вBC). (2000c). Ivory Coast reins in soldiers, $B B C$ News. Recuperado de http://news.bbc.co.uk/2/hi/africa/998144.stm

British Broadcasting Corporation (ввс). (2002). Heavy gun fire in Ivorian city, BBC News. Recuperado de http://news.bbc.co.uk/2/hi/africa/2267971.stm 
British Broadcasting Corporation (ввС). (2003). Ivory Coast: who are the rebels?, BBC News. Recuperado de http://news.bbc.co.uk/2/hi/africa/ 2662655.stm

British Broadcasting Corporation (ввC). (2003a). Ivorian rebels stick to peace deal, $B B C$ News. Recuperado de http://news.bbc.co. uk/2/hi/africa/2724805.stm

British Broadcasting Corporation (вBC). (2003b). Ivorian peace deal provokes fury, $B B C$ News. Recuperado de http://news.bbc.co.uk/2/hi/africa/2715779.stm

British Broadcasting Corporation (ввс). (2004a). France attacks Ivorian airbase, BBC News. Recuperado de http://news.bbc.co.uk/2/hi/africa/ 3988769.stm

British Broadcasting Corporation (ввC). (2006). PM signals new Ivorian stand-off, $B B C$ News. Recuperado de http://news.bbc.co.uk/2/hi/africa/6130248.stm

Brubaker, R. (1992). Citizenship and Nationhood in France and Germany. Cambridge, MA: Harvard University Press.

Chirot, D. (2006). The debacle in Côted'Ivoire. Journal of Democracy, 17(2), 63-77.

Collier, P. y Hoeffler, A. (2004). Greed and grievance in civil war. Oxford Economic Papers, 56(4), 563-595.

Colombant, N. (2006). Gbagbo speech divides Ivorians, Voice of America. Recuperado de http://www.voanews.com/english/archive/2006-12/2006-12-20-voa26. cfm?moddate $=2006-12-20$

Dorman, S., Hammett, D. y Nugent, P. (Eds.). (2007). Making Nations, Creating Strangers: States and citizenship in Africa. Leiden: Brill.

Dorman, S., Hammett, D. y Nugent, P. (2007). Making Nations. En M. Bøås y K. Dunn (Eds.), African Guerrillas: Raging against the machine. Boulder, co: Lynne Rienner Publishers. 
Dozon, J. P. (2000). La Côte d'Ivoire entre démocratie, nationalisme et ethnonationalisme. Politique Africaine, 78, 45-62.

Epstein, D. (2008). International involvement in Côte d'Ivoire. Global Policy Forum. Recuperado de http://www.globalpolicy.org/component/content/article/191/38521.html

Geschiere, P. (2009). The Perils of Belonging: Autochthony, citizenship, and exclusion in Africa and Europe. Chicago, IL.: University of Chicago Press.

Gobierno de Costa de Marfil. (2009, noviembre 29). Conseil des Ministres: L'election presidentielle fixee au 29 novembre 2009. Portail Officiel du Gouvernement de Cote d'Ivoire. Recuperado de http://www.gouv.ci/_actualite-article.php?recordID=690

Goulding, M. (1999). The United Nations and conflict in Africa since the Cold War. African Affairs, 98(391), 155-166.

International Crisis Group. (2003). Côte d'Ivoire: “The War Is Not Yet Over”. ICG Africa Report, 72.

International Crisis Group. (2005). Côte d'Ivoire: Les demi-mesures ne suffiront pas. Briefing Afrique de Crisis Group, 33.

Koulibaly, M. (2003). La Guerre de la France contre la Côte d'Ivoire. La Refondation, Abidjan. París: Harmattan.

Langer, A. (2008). Horizontal inequalities and violent conflict: Côte d'Ivoire country paper. Human Development Report, UNDP. Recuperado de http://hdr.undp.org/docs/publications/background_papers/2005/HDR2005_Langer_Arnim_32.pdf

Linz, J. y Stepan, A. (1996). Problems of Democratic Transition and Consolidation: Southern Europe, South America, and post-Communist Europe. Baltimore, MD: Johns Hopkins University Press. 
Mamdani, M. (1996). Citizen and Subject: Contemporary Africa and the legacy of late colonialism. Princeton, NJ: Princeton University Press.

Marshall, T. H. (1964). Class, Citizenship, and Social Development: Essays. Doubleday. Garden City, NY: Doubleday.

Marshall-Fratani, R. (2006). The war of "who is who": autochthony, nationalism, and citizen- ship in the Ivoirian crisis. African Studies Review, 49(2), 9-43.

Mehler, A. (2009). Peace and power sharing in Africa: a not so obvious relationship. African Affairs, 108(432), 453-473.

Nandjui, P. (1995). Houphouët-Boigny: L'homme de la France en Afrique. Paris: L'Harmattan.

Nhema, A. y Zeleza, T. (eds.). (2008). The Roots of African Conflicts: The causes and costs. Oxford; Athens, Ohio; Pretoria: James Currey; Ohio University Press; Unisa Press.

Nyamnjoh, F. (2006). Insiders and Outsiders: Citizenship and xenophobia in contemporary Southern Africa. Dakar: Zed Books Ltd.

Oficina del Primer Ministro, República de Costa de Marfil. (2008, marzo 6). Communique du Porte-Parole du Premier Ministre (No. 01/050308). Recuperado de http:// www.audiencesforaines.gouv.ci/pdf/Communique_4eme_Reunion_Gpe_de_travail_ AudiencesForaines_5mars2008.pdf

Reno, W. (1999). Warlord Politics and African States. Boulder, co: Lynne Rienner Publishers.

República de Costa de Marfil. (s. f.). Loi n. ${ }^{\circ}$ 61-416 du 14 Décembre 1961 Portant Code de la Nationalité Ivoirienne, modifiée par la loi n. ${ }^{\circ} 72-852$ du 21 décembre 1972.

República de Costa de Marfil. (2007, noviembre). Foire aux Questions. Audiences Foraines. Recuperado de http://www.audiencesforaines.gouv.ci/foire.php 
Richards, P. (2005). To fight or to farm? Agrarian dimensions of the Mano River conflicts (Liberia and Sierra Leone). African Affairs, 104(417), 571-590.

Smith, S. (2003). La politique d'engagement de la France àl 'épreuve de la Côte d'Ivoire. Politique Africaine, 89, 112-126.

Soro, G. (2005). Pourquoi je suis devenu un rebelle: La Côte d'Ivoire au bord du gouffre; entretiens avec Serge Daniel. Paris: Hachette Littératures.

Toungara, J. (2001). Ethnicity and political crisis in Côted'Ivoire. Journal of Democracy, 12(3), 63-72.

un Security Council. (2007). Twelfth progress report of the Secretary-General on the United Nations operation in Côte d'Ivoire (S/2007/133, un, Nueva York, 8 de marzo de 2007).

UN Security Council. (2009). Nineteenth progress report of the Secretary-General on the United Nations operation in Côte d'Ivoire (S/2009/21, un, Nueva York, 8 de enero de 2009).

Widner, J. (1994). Two leadership styles and patterns of political liberalization. African Studies Review, 37(1). 151-174.

Woods, D. (2003). The tragedy of the cocoa pod: rent-seeking, land and ethnic conflict in Ivory Coast. Journal of Modern African Studies, 41(4), 641-655.

Zartman, I. W. (ed.). (1995). Collapsed States: The disintegration and restoration of legitimate authority. Boulder, co: Lynne Rienner Publishers. 